\title{
Méthode des coefficients de réaction appliquée en France pour les calculs des soutènements : influence de la variation des paramètres"
}

\author{
A. DHOUIB \\ GEO-SIGMA, Paris
}

On rappelle I'analogie de comportement entre un ressort " parfaitement élastique $»$ (modèle de Winkler) et un sol (cpseudo-élastique ») pour expliquer la notion du coefficient de réaction du sol utilisée dans le calcul et le dimensionnement des soutènements en France.

On étudie, à partir de calculs numériques basés sur la méthode aux coefficients de réaction de type “ DENEBOLA-LPC », I'incidence du coefficient de réaction horizontal $k_{h}$, des paramètres de cisaillement $c$ et $\varphi$ et de la rigidité El de la paroi de soutènement sur l'évolution théorique des déplacements et des pressions d'une paroi tirantée et d'une paroi autostable.

\section{Methods based on subgrade reaction coefficients used in France to design retaining walls : parameter study}

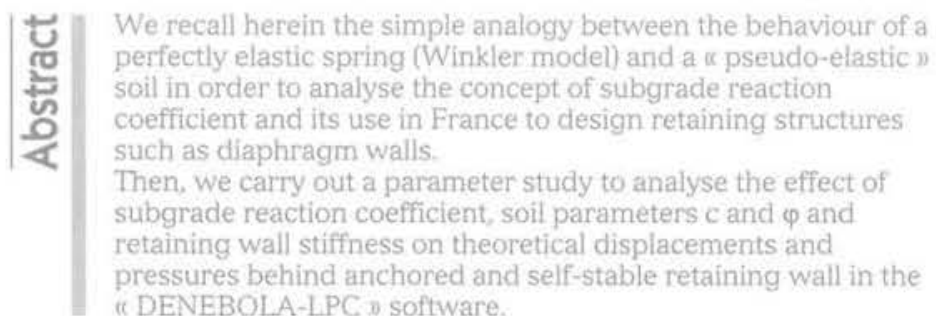

- La présente communication fait partie de l'ensemble des conférences présentées, le 14 septembre 1994 , au Comité français de mécanique des sols. Elle est la suite des articles regroupés dans la Revue françaisé de géotechnique $n^{\circ} 71$ du $2^{\prime \prime}$ trimestre 1995. 


\section{NOTATIONS}

a

B

largeur d'un massif enterré soumis à des efforts de renversement.

c : cohésion du sol,

C. : terme de cohésion lié à la pression limite de poussée,

$c_{0}$; cohésion de référence $\left(c_{0}=30 \mathrm{kPa}\right)$

$C_{p}$ : terme de cohésion lié à la pression limite de butée,

El : rigidité d'une paroi d'épaisseur e,

$\mathrm{E}_{\mathrm{M}}$; module de déformation pressiométrique Ménard,

$E_{5}$ : raideur du sol $\left(=2\right.$ à $\left.3 E_{M}\right)$,

$\varphi \quad$ : angle de frottement interne du sol,

$\gamma \quad$ : poids volumique du sol,

h : hauteur du même massif enterré soumis à des efforts de renversement,

h. : hauteur caractéristique qui définit l'étendue de la zone de contact effectif sol-structure,

$K_{3} \quad$ : coefficient de pression limite de poussée,

$\mathrm{k}_{\mathrm{h}}$ : coefficient de réaction horizontal du sol (analogue à un poids volumique),

$k_{\mathrm{he}}$ : coefficient de réaction coté de la poussée,

$k_{h \max }$ : coefficient de réaction à T'origine de la courbe " pression-déplacement »,

$k_{\text {hp }}$ : coefficient de réaction coté de la butée,

$\mathrm{K}$ : coefficient des terres au « repos ») (conditions œdométriques),

$\mathrm{K}_{\mathrm{p}} \quad$ : coefficient de pression limite de butée,

$k_{\mathrm{v}} \quad$ : coefficient de réaction vertical du sol (analogue à un poids volumique),

$\mathrm{p}_{\mathrm{a}} \quad$ : pression limite de poussée,

$\mathrm{p}_{\mathrm{p}}$ : pression limite de butée,

th : tangente hyperbolique,

$\mathrm{u}$ : déplacement horizontal dũ à une pression horizontale $\mathrm{p}_{\mathrm{h}^{\prime}}$

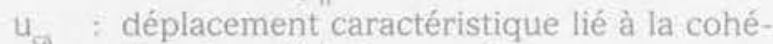
sion du coté de la poussée,

$u_{c p}$ : déplacement caractéristique lié à la cohésion du coté de la butée,

$u_{00} \quad$ : déplacement caractéristique lié à l'angle de frottement du coté de la poussée,

$\mathrm{u}_{\mathrm{op}} \quad$ : déplacement caractéristique lié à l'angle de frottement du côté de la butée.

w : déplacement vertical dû à une pression verticale p.

1

\section{Notion physique, analogie de comportement mécanique}

\section{1}

\section{Cas du ressort}

Lorsqu'une force ponctuelle P est appliquée à l'extrémité d'un ressort de raideur (ou coefficient de rappel) $k$, celui-ci subit un allongement (u) ou un raccourcissement $(-\mathrm{u})$. La relation élémentaire qui relie, dans le domaine élastique linéaire, les divers paramètres est :

$$
\mathrm{P}=\xi \xi \mathrm{k} \cdot \mathrm{u},
$$

$\xi$ vaut +1 lorsque le ressort est en traction simple, - 1 pour la compression simple. Une telle relation représente le modèle classique de Winkler (1868).

Ce modèle montre que (Figure 1.a) :

- lorsque la raideur $k$ du ressort augmente, le déplacement $u$ diminue pour le même effort ponctuel appliqué P:

- une augmentation de la force $\mathrm{P}$ au-delà de la résistance caractéristique de rupture $\mathrm{P}$. du ressort conduit à la rupture de ce dernier : les déplacements u ne dépendent plus, à ce stade de déformations, de la raideur $k$ et tendent vers des valeurs $\alpha$ infinies $)$;

- la raideur $k$ du matériau du ressort régit donc le domaine d'élasticité linéaire et limite, par conséquent, la plage de variations des déplacements (allongement ou raccourcissement) d'origine élastique. En effet, plus le ressort est rigide, plus les déplacements, avant rupture, sont faibles et la plastification rapidement atteinte.

1.2

\section{Analogie sol-ressort}

Dans les logiciels de calculs automatiques récents et particulièrement les plus utilisées en France ( $\propto$ DENEBOLA-LPC », « RIDO ) et ( PAROI )), faisant appel à la notion du coefficient de réaction, on introduit un modèle analogue à celui de Winkler (Fig. 1.b). Le sol exerce (cas de parois de soutènement, Fig. 1.c) ou " subit $»$ (cas de massif de fondation enterré, Fig. 1.d) une pression surfacique p respectivement sur ou par la structure de contact qui soit se déplace de u soit tasse de w.

Avant la mobilisation des pressions limites du sol au contact d'une paroi verticale (pressions active coté poussée $p_{\text {, }}$ ou passive coté butée $p_{\text {p }}$ ), la pression $p$ est liée, dans le domaine "pseudo-élastique », par une équation analogue à la relation (1), soit :

$$
\mathrm{p}=\mathrm{p}_{\mathrm{o}}+\xi \cdot \mathrm{k}_{\mathrm{s}} \cdot \mathrm{u}_{\mathrm{t}}
$$

$\mathrm{k}_{\mathrm{s}}$ est le coefficient de réaction du sol, analogue à un poids volumique,

$\mathrm{p}_{\mathrm{o}}$ désigne la pression qui règne dans le sol avant sa mise en charge ou en décharge,

$\xi$ permet de tenir compte du mode de sollicitation entre le sol et la paroi de contact $(\xi=-1$ coté poussée, et $\xi=+1$ coté butée).

En conclusion, l'analogie « sol-ressort » montre que :

- le coefficient de réaction du sol régit les déplacements qui permettent de mobiliser les pressions limites de poussée et de butée du sol. Plus le sol est raide, plus les déplacements sont faibles; le domaine « pseudo-élastique $n$ se réduit, l'état limite de poussée et/ou de butée est atteint ;

- la mobilisation complète des pressions limites conduit à la rupture du sol et à des déplacements « importants» où le coefficient de réaction $\mathrm{k}_{\mathrm{s}} \mathrm{du}$ sol ne joue, à ce niveau de déformations, aucun rôle. 


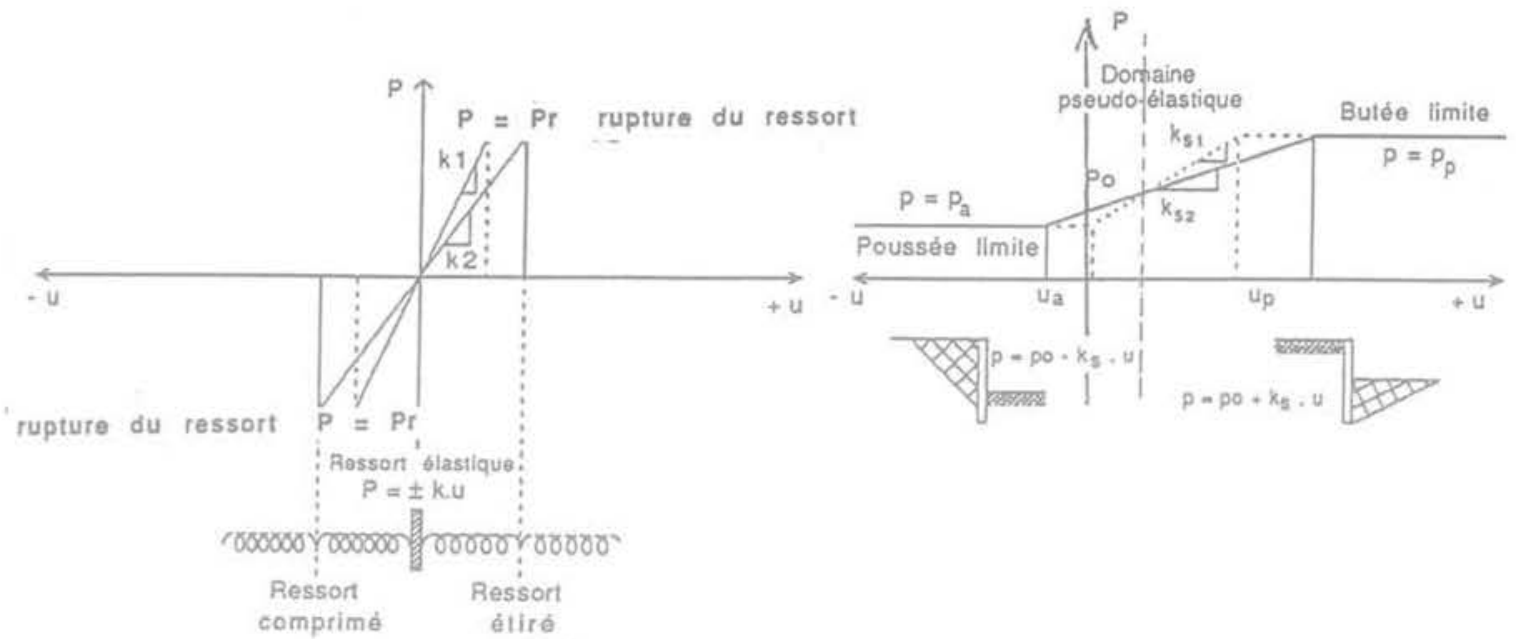

a- comportement du ressort

b- Comportement élastique-parfaitement plastlque de poussée-butée du sol

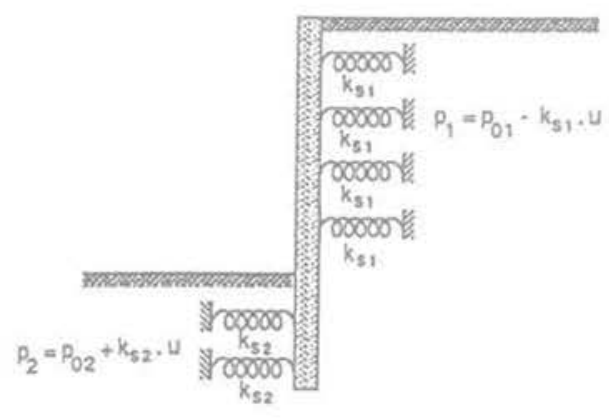

c- Cas de parol de la soutènement

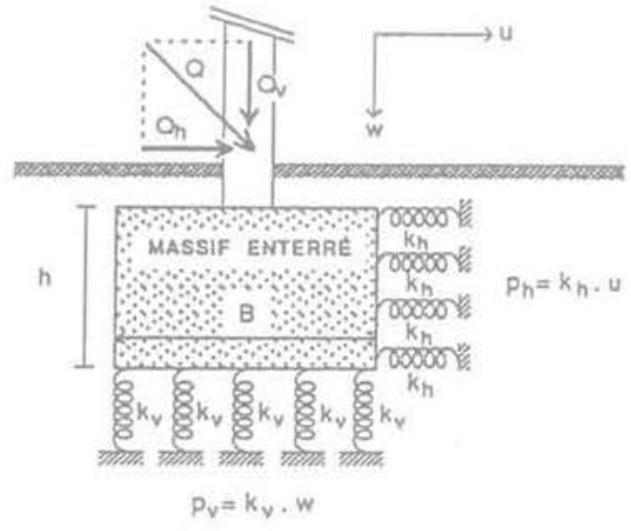

d- Cas du massif enterré

\section{3}

\section{Application aux parois verticales de soutènement}

\section{$1,3.1$}

\section{Formulation de type Ménard et Bourdon}

Par application de la formulation de L. Ménard et C. Bourdon (1964), J. Balay (1985) a fixé, dans le calcul du coefficient de réaction horizontal $\mathrm{k}_{h}$, les valeurs d'un paramètre dimensionnel a (analogue a la hauteur h de la semelle de la figure 1.d). En l'absence d'éléments ponctuels de confortement de la paroi verticale de soutènement (tirants d'ancrage, butons), une telle formulation conduit à l'expression suivante :

$$
k_{h}=\frac{E_{M}}{\frac{6}{5} \cdot a \cdot\left[\frac{2}{5} \cdot \alpha+(9 \cdot a)^{\alpha-1}\right]}
$$

A la lumière de ce résultat, il y a lieu de noter que:

- une telle formulation de $k_{h}$ est compatible avec l'idée que le coefficient de réaction n'est pas un paramètre intrinsèque du sol. En effet, ce coefficient dépend, en plus du module pressiométrique Ménard $E_{M}$ et du coefficient rhéologique $\alpha$ du sol ambiant, de la géométrie de la paroi de soutènement;

- la modélisation ainsi proposée respecte la continuité des déplacements normaux (perpendiculaires à la fibre neutre de la paroi de soutènement). Elle peut intégrer aussi de façon empirique à ce jour le mécanisme de « décollement » par la prise en compte d'un «mortterrain m lorsque la hauteur a de la paroi est supérieure à la hauteur caractéristique a. (Monnet, 1994). Mais contrairement à ce que l'on pense, une telle modélisation ne constitue pas une loi d'interaction sol-paroi car elle n'introduit pas le mode de glissement qui peut se produire entre le sol et la paroi de soutènement. L'introduction du mécanisme de glissement est possible en associant, par exemple, aux ressorts « normaux 》 des éléments de rigidité tangentiels (ressorts 
parallèles à la face de contact sol-structure), comme dans la modélisation d'interfaces par éléments finis (A. Barbas et R. Frank, 1982). Cependant, une telle procédure ne paraît pas pouvoir améliorer la méthode actuelle de calcul faisant appel aux coefficients de réaction.

Notons enfin que dans ce cadre, la présente formulation pose quelques problèmes : lorsque le paramètre dimensionnel a augmente considérablement, $\mathrm{k}_{\mathrm{h}}$ diminue fortement. Inversement, ce dernier est très élevé pour des valeurs de a très faibles et ce pour un même sol au contact d'une même paroi ou au contact de parois à rigidités différentes.

Ce constat a conduit A. Monnet (1994) à lier $\mathrm{k}_{\mathrm{h}}$ à la rigidité de la paroi de soutènement tout en s'inspirant de l'abaque interne et non publié de Chadeisson.

\section{6}

\section{Approche de type A. Monnet}

La formulation de A. Monnet est basée sur une démarche intéressante pour prôner la validité de l'abaque de Chadeisson. Cette démarche tient compte, d'une manière implicite, de la géométrie de la paroi en la liant directement à la rigidité (El) du soutènement et aux paramètres de cisaillement ( $c$ et $\varphi$ ) du sol de contact en butée avec la paroi, soit:

$$
k_{h p}=\beta_{p} \cdot\left[\frac{120}{11} \cdot \operatorname{El} \cdot\left(\frac{K_{p} \cdot \gamma}{u_{\varphi p}}\right)^{4}\right]^{1 / 5}+\frac{C_{p} \cdot c^{\prime} \cdot \text { th }\left(\frac{c^{\prime}}{c_{0}}\right)}{u_{c p}}
$$

avec :

$$
\beta_{p}=\left(1-\frac{K_{0}}{K_{p}}\right)
$$

Il y a lieu de noter que l'introduction de la rigidité de la paroi dans l'estimation de $\mathrm{k}_{\text {}}$ a été également faite par J.-P. Gigan (1984) à partir des résultats de calculs théoriques par élérnents finis effectués par S. Barussaud (1981) pour l'étude de l'interaction sol-paroiancrage. Contrairement à A. Monnet, J.-P. Gigan faisait intervenir la raideur $\mathrm{E}_{\mathrm{s}}\left(=2\right.$ à $\left.3 \mathrm{E}_{\mathrm{M}}\right)$ du sol et implicitement la rigidité de la paroi au moyen de la longueur de transfert 1 qui traduit la rigidité relative sol-paroí.

En effet, la formulation de $k_{n}$ en fonction de la ridigité de la paroi de soutènement est, à notre avis, indiscutable : une paroi rigide conduit à une augmentation des contraintes derrière la paroi et à une diminution des déplacements de la paroi rigide. Une telle augmentation de la pression ne compense pas la diminution des déplacements. Le coefficient de réaction $\mathrm{k}_{\mathrm{h}}$ ne peut qu'augmenter. Néanmoins, l'examen des hypothèses multiples, bases de l'approche abordée par A. Monnet (1994), appelle les remarques suivantes :

- la formulation de $k_{h}$ ne peut être valable que si la fiche $\mathrm{h}$ de la paroi en contact avec le sol est inférieure à la hauteur caractéristique $\mathrm{h}_{c}$;

- l'évaluation de $k_{h}$ repose sur l'idée que la compacité du sol reste homogène sur la hauteur $h$ ou $h_{c}$;

- le choix des déplacements caractéristiques rattachés aux paramètres de cisaillement (c et $\varphi$ ) est arbitraire. Ceci vaut également pour le choix du terme de cohésion intervenant dans la forme finale de $\mathrm{k}_{\mathrm{h}}$.
Une démarche analogue à celle de A. Monnet conduit à exprimer le coefficient de réaction $k_{h}$ du sol en poussée par :

$$
k_{\text {ha }}=\beta_{a} \cdot\left[30 \cdot E l \cdot\left(\frac{K_{a} \cdot \gamma}{u_{p a}}\right)^{4}\right]^{1 / 5}+\frac{C_{a} \cdot c^{\prime} \cdot \operatorname{th}\left(\frac{c^{\prime}}{c_{0}}\right)}{u_{c a}}
$$

avec :

$$
\beta_{\mathrm{a}}=\left(\frac{\mathrm{K}_{0}}{\mathrm{~K}_{\mathrm{a}}}-1\right)
$$

L'analyse de A. Monnet repose, comme toutes les approches récentes basées sur le coefficient de réaction, sur l'hypothèse de comportement «pseudo-élastique o linéaire du sol entre les deux états limites de pressions en poussée active et en butée passive connue sous le nom de « loi élasto-plastique » (Fig. 1.b). L'introduction d'une loi de réaction hyperbolique des $\alpha$ pression-déplacements » (Kondner, 1964) conduit à une expression analogue à l'égalité (2), soit (Fig. 2) :

$$
p=p_{0}+\xi \cdot \frac{u}{\left(\frac{1}{k_{h \max }}+\frac{u}{p_{u}}\right)}
$$

$k_{\text {himax }}$ désigne la valeur maximale du coefficient de réaction à l'origine de la courbe hyperbolique de réaction et $\mathrm{p}_{u}$ la pression limitant la courbe de réaction $\left(=\mathrm{p}_{\mathrm{a}}\right.$ ou $\left.p_{p}\right)$. $\xi$ garde la même définition que dans l'équation (2).

Une approche similaire à celle de A. Monnet conduit à une formulation des coefficients de réaction analogue à celle donnée par les égalités (3) et (4) précédentes mais en remplaçant les valeurs de $\beta$ par :

$$
\beta_{p}=\frac{K_{p}}{K_{0}} \cdot\left(1-\frac{K_{0}}{K_{p}}\right) \quad \text { (côté butée), }
$$

et

$$
\beta_{a}=\frac{\left(\frac{K_{0}}{K_{a}}-1\right)}{\left(2-\frac{K_{0}}{K_{a}}\right)} \quad \text { (côté poussée). }
$$

Les coefficients de réaction obtenus à partir des identités (3a) et (4a) constituent des valeurs maximales (pente à l'origine de la courbe de réaction) où l'on peut lier la pression de rupture $\mathrm{p}_{\mathrm{u}}$ à un déplacement caractéristique $\mathrm{u}_{\mathrm{o}}$ par :

$$
\mathrm{p}_{\mathrm{u}}=\mathrm{k}_{\operatorname{minax}} \cdot \mathrm{u}_{\mathrm{o}}
$$

La combinaison des égalités (5) et (6) conduit à un coefficient de réaction $k_{h}$ qui décroit en fonction des déplacements croissants u de la paroi et dont la valeur est donnée par l'équation hyperbolique :

$$
\mathrm{k}_{\mathrm{h}}=\frac{\mathrm{k}_{\mathrm{hmax}}}{\left(1+\frac{\mathrm{u}}{\mathrm{u}_{0}}\right)}
$$

Ce résultat concorde parfaitement avec les observations de L. Ménard (1962) qui précise que les coeffi- 


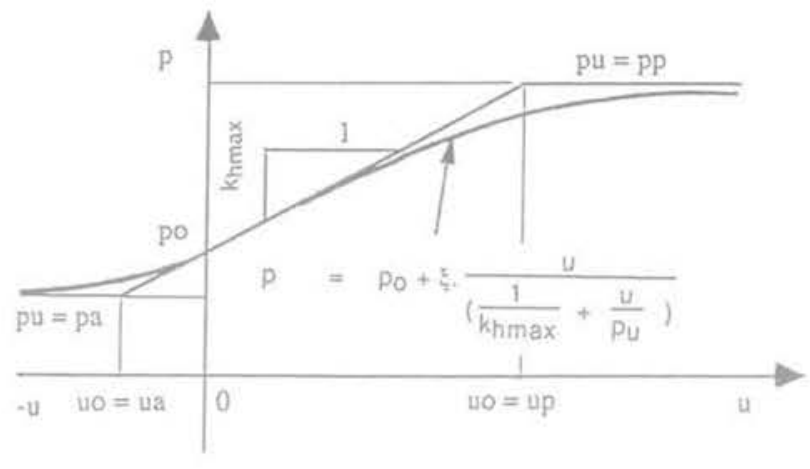

FG:2 Loi de réaction hyperbolique pour le calcul des coefficients de réaction. Subgrade reaction coefficient ; hyperbolic reaction rule.

cients de réaction, correspondant à de très faibles déplacements dans la phase purement élastique, sont nettement plus élevés que ceux de la phase «pseudoélastique .. En effet, les coefficients de réaction dépendent du taux de déplacements atteint :

- ils sont maximaux à l'origine de la courbe de réaction, ce qui correspond, en pratique, soit aux premières phases de terrassement soit à la mise en tension de tirants d'ancrage limitant les déplacements ;

- ils diminuent progressivement lorsque les déplacements augmentent soit en l'absence de tirants d'ancrage soit dans les dernières phases de terrassement.

Dans le but d'apprécier le rôle du coefficient de réaction $\mathrm{k}_{\mathrm{h}}$, des caractéristiques de cisaillement c et $\varphi$ des sols et enfin de la rigidité de la paroi, nous présentons, dans ce qui suit, les résultats de calculs theoriques effectués par " DENEBOLA-L.PC », pour deux types de paroi de soutènement : paroi tirantée et paroi autostable.

Il convient de préciser que l'étude paramétrique présentée ici n'a pas pour objet de comparer les différentes approches actuelles de calcul du coefficient de réaction $\mathrm{k}_{\mathrm{b}}$. Elle est juste destinée à montrer l'incidence des différents paramètres.

\section{2 \\ Étude paramétrique Influence des divers paramètres}

\section{1}

\section{Cas d'une paroi tirantée}

La paroi étudiée présente une épaisseur de $0.4 \mathrm{~m}$, comporte 2 lits de tirants (respectivement à $1,5 \mathrm{~m}$ et à $6,5 \mathrm{~m}$ ) et est ancrée dans une craie relativement saine, le fond de fouille étant à $9,10 \mathrm{~m}$.

L'évolution, en fonction de la profondeur, des déplacements et des pressions résuitantes calculés par «DENEBOLA-LPC » à la dernière phase de terrrassement (phase 4) est présentée sur la figure 3 pour différentes valeurs théoriques du coefficient de réaction $\mathrm{k}_{\mathrm{h}}$.

L'examen de ces diagrammes conduit à noter les points suivants :

- les déplacements ne diminuent pas proportionnellement aux coefficients de réaction. Plus la valeur de $k_{h}$ est élevée, plus la diminution des déplacements $u$ est faible : en effet, une augmentation importante de $\mathrm{k}_{\mathrm{h}}$ conduit à « rigidifier ») le milieu et à obtenir rapidement les états limites de poussée et de butée généralisés derrière la paroi de soutènement :

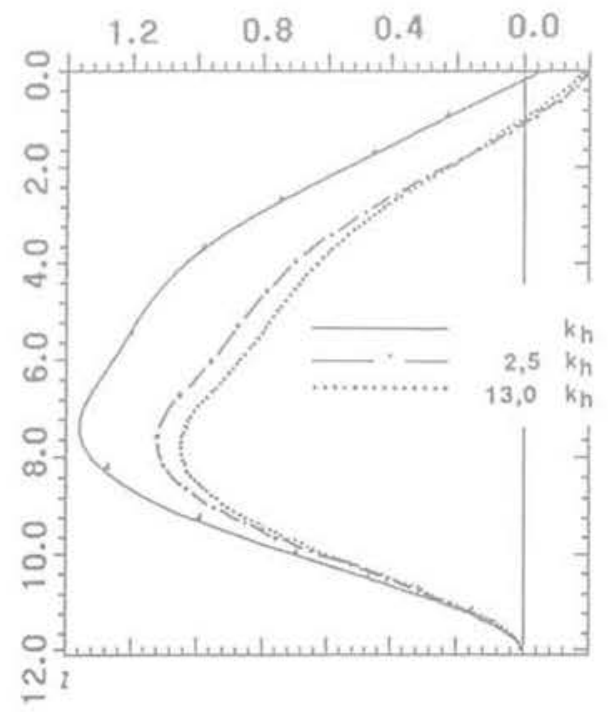

a- déplacements $\left(10^{-2} \times \mathrm{m}\right)$

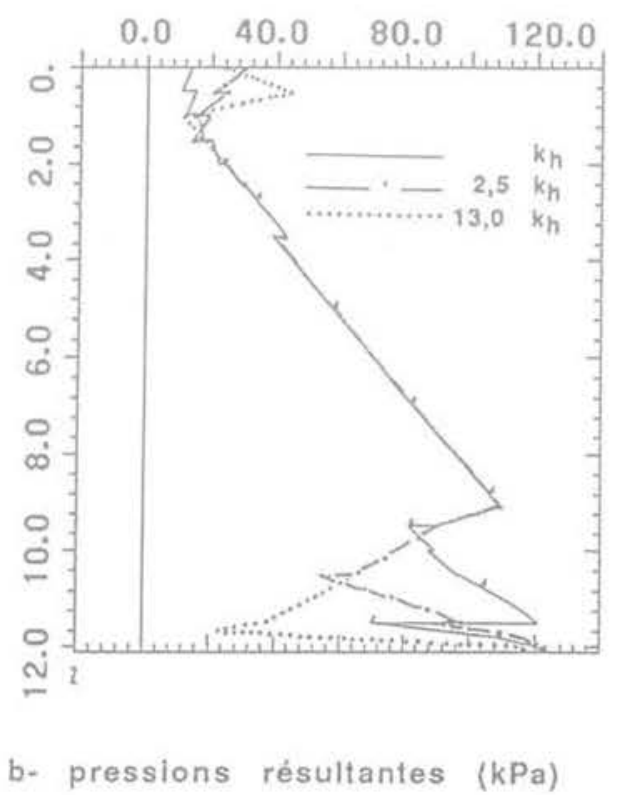

FIG:3 Influence du coefficient de réaction : cas d'une paroi avec deux lits de tirants d'ancrage, Effect of subgrade reaction coefficient variation : anchored retaining wall case, 
- les pressions résultantes sont affectées en tête (zone d'influence du premier tirant) et en particulier dans la partie en fiche de la paroi où s'étend une « plastification » progressive lorsque $\mathrm{k}_{\mathrm{h}}$ augmente ;

- les efforts internes (efforts tranchants et moments fléchissants) dans la paroi subissent des variations minimes qui ne suivent pas les déplacements.

L'étude de l'incidence des autres paramètres conduit à noter les points suivants :

- une légère augmentation de la cohésion conduit à une diminution des déplacements. Cette diminution est relativement faible et devient nulle pour une valeur caractéristique de la cohésion à partir de laquelle les déplacements, les pressions et les efforts internes (efforts tranchants et moments fléchissants) se stabilisent;

- les valeurs croissantes de l'angle de frottement interne du sol (variant entre 25 et $45^{\circ}$ ) conduisent à des variations décroissantes et globalement quasi linéaires des déplacements, les pressions et les efforts internes ne subissent que de faibles variations localisées;

- l'augmentation de la rigidité propre de la paroi conduit surtout à modifier le mode de déplacement de la paroi : lorsque la rigidité augmente et les efforts dans les tirants sont maintenus constants, les déplacements diminuent dans la partie centrale de la paroi, mais à partir d'une valeur caractéristique de rigidité, les déplacements augmentent en tête de la paroi correspondant à un mode de déplacement d'une paroi rigide.

\section{2}

\section{Cas d'une paroi autostable}

Il s'agit d'une paroi autostable de 0,80 m d'épaisseur qui retient un sol homogène sur une puissance de $8 \mathrm{~m}$ et d'une fiche de $4 \mathrm{~m}$ (première phase de terrassement).
L'étude paramétrique effectuée ici porte sur l'influence des paramètres suivants :

- coefficients de réaction $\mathrm{k}_{\mathrm{h}}$;

- caractéristiques de cisaillement du sol (c et $\varphi$ ) ;

- la rigidité propre El de la paroi.

\subsection{1}

\section{Effet du coefficient de réaction $\mathrm{k}_{\mathrm{h}}$}

Les calculs théoriques effectués, dans ce cadre, montrent que (Fig. 4) :

- si l'augmentation de $\mathrm{k}_{\mathrm{h}}$ entraîne une diminution des déplacements de la paroi dans un rapport non linéaire, contrairement à la paroi tirantée, on ne note pas de stabilisation « rapide $)$ des déplacements;

- toute forte augmentation de $\mathrm{k}_{\mathrm{p}}$ conduit à limiter, comme pour la paroi tirantée, le domaine «pseudoélastique » et à accélérer la « plastification » progressive de la paroi autostable;

- pour des coefficients de réaction croissants, les pressions résultantes subissent, la hauteur libre étant en état limite de poussée, des variations décroissantes dans la zone en fiche. Cette diminution devient plus faible pour des valeurs plus élevées du coefficient de réaction $\mathrm{k}_{\mathrm{h}}$.

\section{2:2}

\section{Incidence des caractéristiques de cisaillement c et $\varphi$}

La cohésion paraît le paramètre le plus influent sur l'évolution théorique des déplacements et des pressions de la paroi autostable (cf. Figure 5.a et b) : en effet, une légère augmentation de la cohésion conduit à une forte diminution des déplacements et des pressions. Mais, cette diminution est limitée à une cohésion
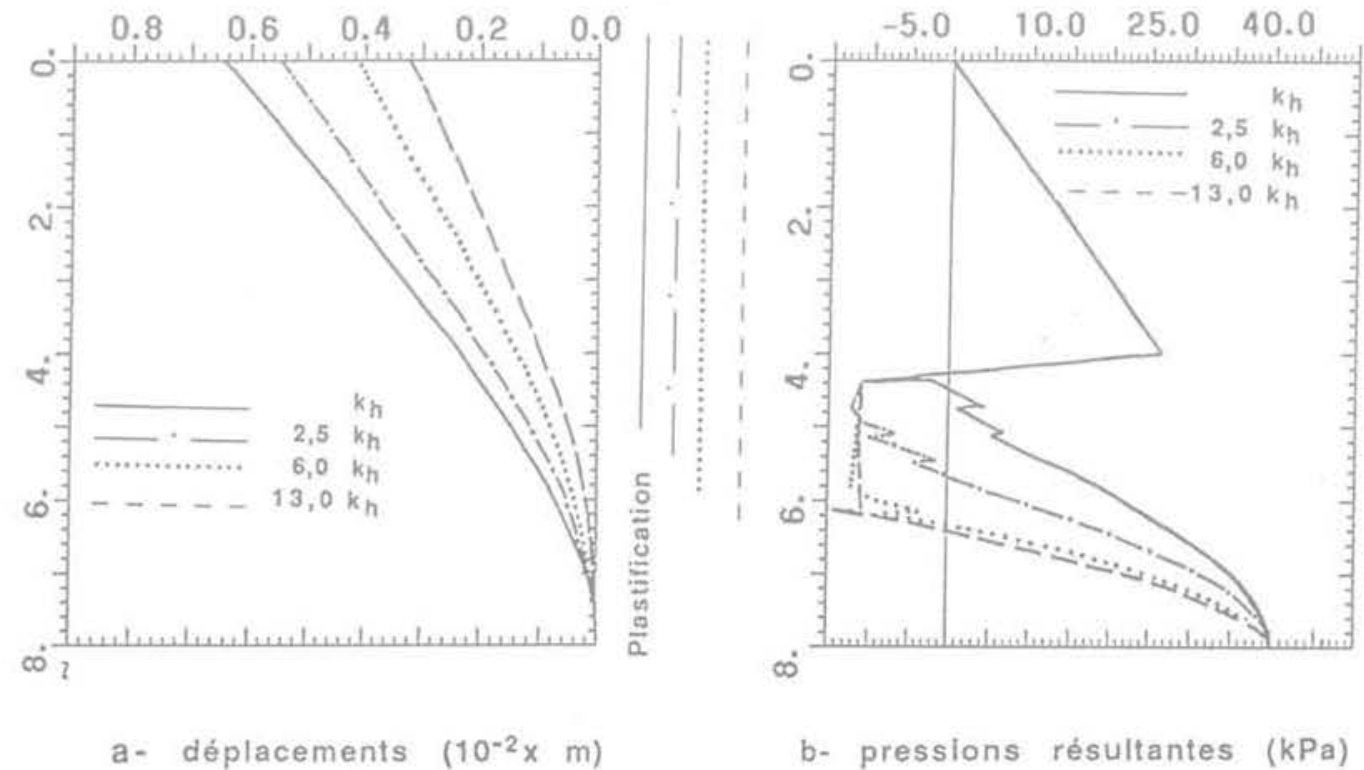

b- pressions résultantes ( $\mathrm{kPa})$ 

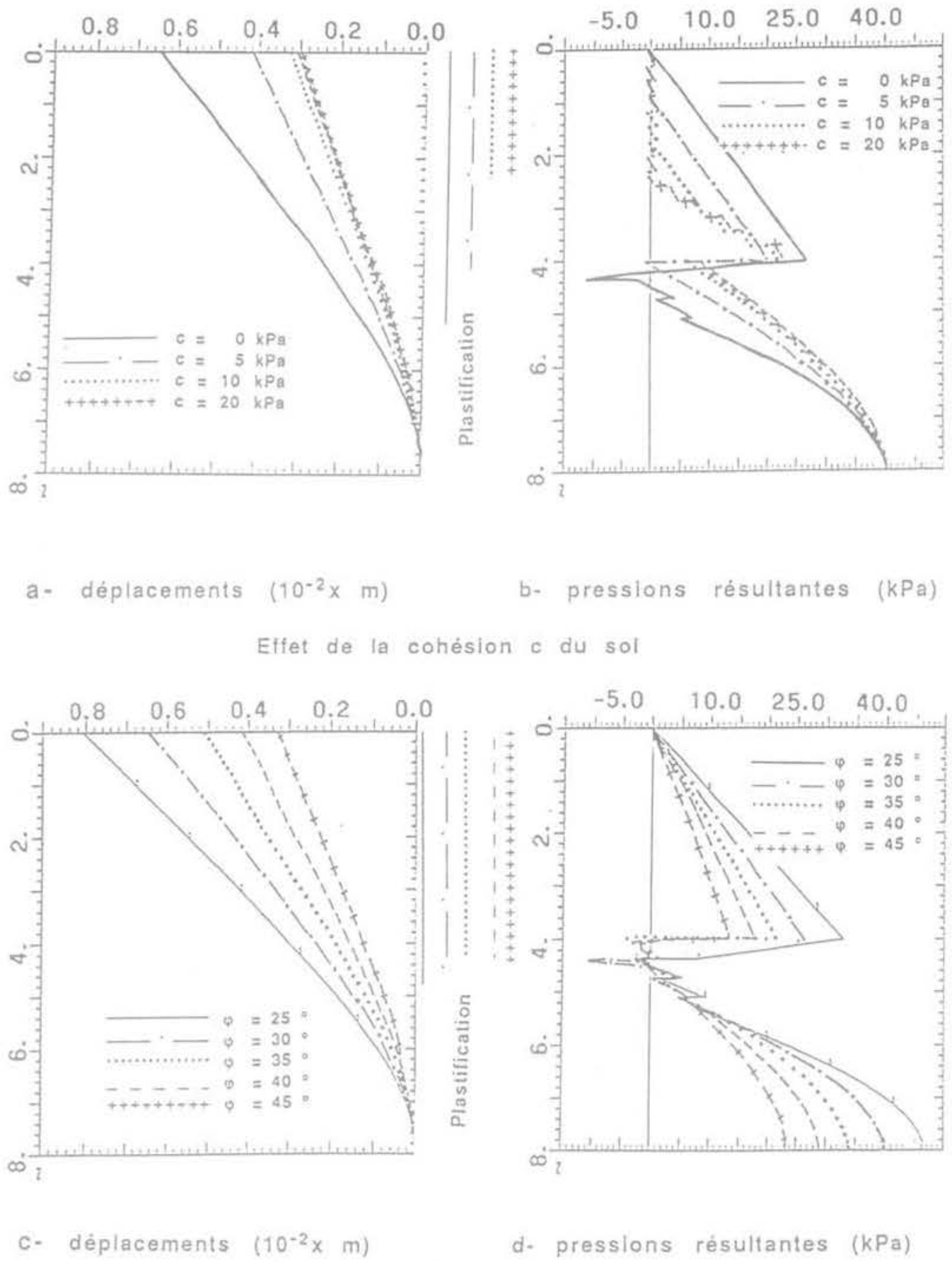

c- déplacements $\left(10^{-2} \times \mathrm{m}\right) \quad$ d- pressions résultantes (KPa)
Effet de l'angle de frottement propre $\varphi$ du sol

FG.5. Paroi autostable : influence des paramètres géotechniques c et $\varphi$. Self-stable retaining wall : effect of the variation of geotechnical parameters $\mathrm{c}$ and $\mathrm{\varphi}$.

caractéristique à partir de laquelle le domaine ( pseudo-élastique » est stabilisé et les déplacements et les pressions ne subissent aucune variation.

Associée à la cohésion caractéristique, une rigidité croissante de la paroi conduit à la diminution des déplacements mais à l'augmentation des pressions résultantes. Ces augmentations sont dues à des augmentations des pressions derrière la paroi autostable : elles sont accompagnées d'une réduction progressive du domaine « pseudo-élastique ». Par contre, une augmentation de $k_{h}$ pour la valeur de la cohésion caractéristique conduit à limiter le domaine « pseudo-élastique y) et à diminuer les déplacements de la paroi autostable et les pressions résultantes.
Les valeurs croissantes de l'angle de frottement $\varphi$ (ne dépassant pas les valeurs maximales de $40^{\circ} / 45^{\circ}$ ) conduisent à des variations décroissantes et quasi linéaires des déplacements et des pressions résultantes de la paroi autostable, ce qui peut paraître inattendu (Fig. 5.c et d).

\subsection{3}

\section{Influence de la rigidité propre de la paroi (El)}

L'augmentation de la rigidité de la paroi de soutènement autostable conduit ici à la diminution des 


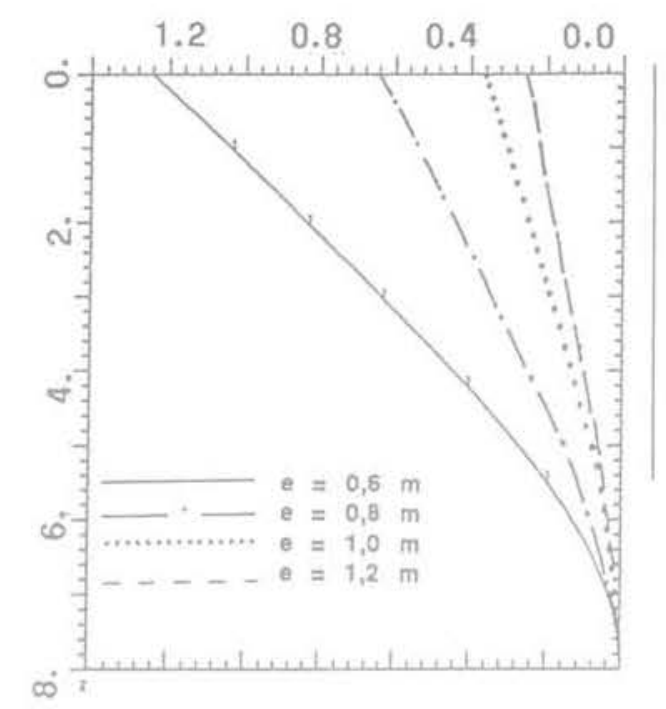

a- déplacements $\left(10^{-2} \times \mathrm{m}\right)$
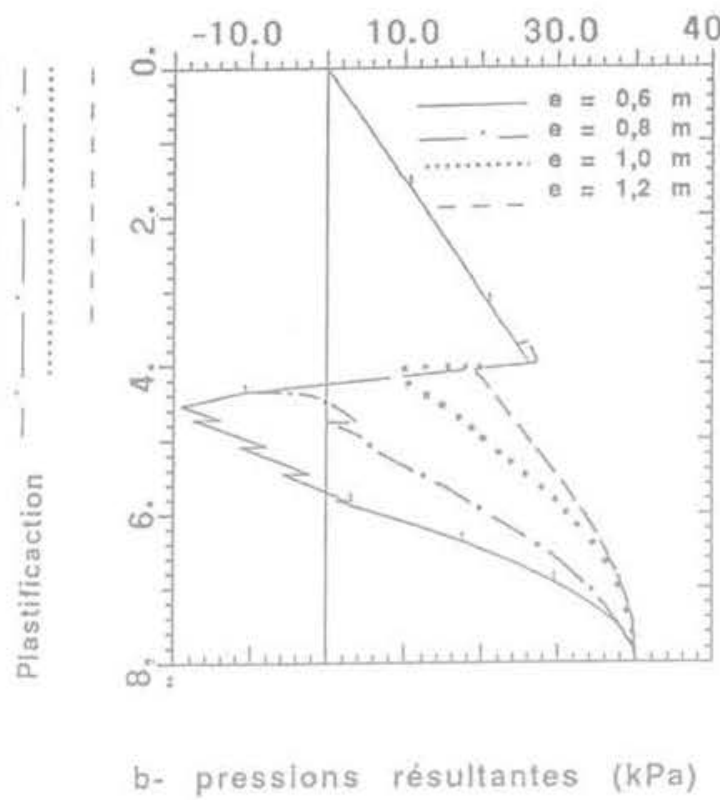

Paroi autostable : influence de la rigidité propre de la paroi.

Self-stable retaining wall : effect of retaining wall stiffiness variation.

déplacements qui tendent vers une stabilisation (Fig.6.a). Cette diminution n'est pas régulière et elle est accompagnée d'une augmentation des pressions en particulier en contre-butée (cf. Fig. 6.b) en raison du mode de déformation de la paroi rigide autostable. En effet, la rigidité propre de la paroi présente, comme la cohésion, une rigidité caractéristique à partir de laquelle les variations des déplacements et des pressions mobilisées sont de plus en plus faibles, voire nulles.

D'une façon générale et selon le mode de déformation de la paroi, toute limitation des déplacements conduit à une augmentation des pressions derrière la paroi. Dans ce cadre, H.F. Schweiger (1994) étudie le comportement d'une paroi de $5 \mathrm{~m}$ de haut en lui imposant des déplacements de translation en poussée et en butée. Les résultats numériques obtenus par l'auteur à partir des modélisations par éléments finis en élastoplasticité sur un sol purement frottant, montrent que : - en comportement de poussée (Fig. 7.a) :

- l'imposition de très faibles déplacements (de $1 \mathrm{~mm}$ dans le présent exemple) de la paroi conduit à des rapports de la pression active $p_{4}$ aux déplacements imposés u élevés (c'est-à-dire du coefficient de réaction),

. l'évolution de $\mathrm{k}(=\mathrm{p} / \mathrm{u})$ croit avec la profondeur dans le milieu granulaire,

- pour des déplacements de translation élevés mais de quelques mm et demeurant inférieurs aux déplacements limites (ici de $7 \mathrm{~mm}$ ), le rapport $\mathrm{k}\left(=\mathrm{p}_{\mathrm{z}} / \mathrm{u}\right)$ décroit rapidement correspondant à un (c relâchement $)$ des grains derrière la paroi ;

- en comportement de butêe (Fig. 7.b), le rapport de la pression passive au déplacement mobilisateur
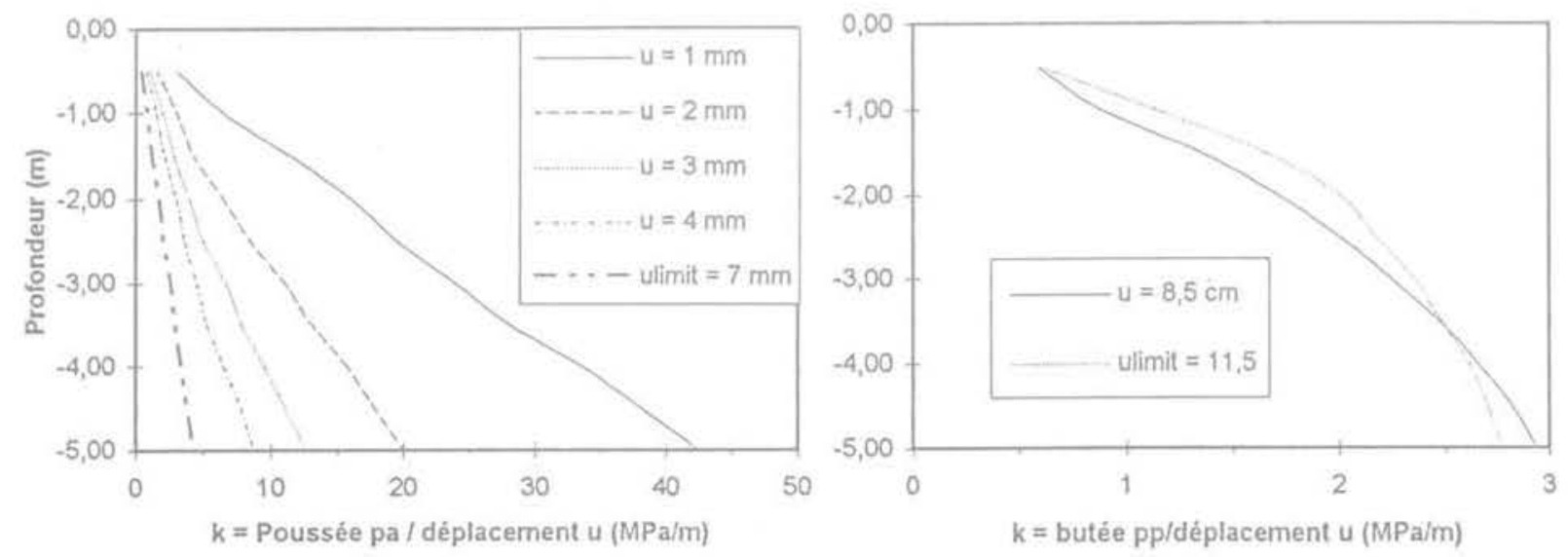

FiG,7 Comportement d'une paroi rigide soumise à un déplacement de translation (Schweiger, 1994). Behaviour of stiff retaining wall submitted to translation. 
imposé croît légèrement dans la partie supérieure de la paroi, puis décroît dans la partie inférieure lorsque les déplacements de translation augmentent. Cette diminution peut s'expliquer par une réorganisation des grains du matériau granulaire plus sollicités en rupture de butée à la base qu'en tête de la paroi. Comparées aux valeurs de $\mathrm{p}_{\mathrm{a}} / \mathrm{u}$ (comportement en poussée) de la figure 7.a, les rapports de $p_{p} / u$ (comportement en butée) de la figure $7 . \mathrm{b}$ sont très faibles. Cette différence peut être due soit aux conditions initiales en déplacements soit à une rupture en butée du matériau granulaire pour les deux niveaux de déplacements imposés qui sont relativement élevés.

La transformation des valeurs obtenues pour les déplacements de poussée de la figure 7.a dans un plan (pression active $\mathrm{p}_{\mathrm{o}}$ /déplacement $u$, déplacement $u$ ) conduisent aux distributions de la figure 8. Comparées, pour différentes profondeurs $\mathrm{z}$, aux calculs numériques par éléments finis (H.F. Schweiger, 1994), les valeurs obtenues à partir de la relation (7) montrent bien que le coefficient de réaction $\mathrm{k}_{\mathrm{b}}$ dépend des déplacements mobilisés derrière la paroi : il est maximal dans le domaine élastique (faibles déplacements) et il décroît, selon une distribution relativement hyperbolique, lorsque les déplacements augmentent. Ce résultat est tout à fait conforme aux observations expérimentales de P. Schmitt (1984) : les variations des déplacements et des pressions résultantes mesurées dans le tirant 112 de la paroi préfabriquée du métro de Lille sont représentées sur la figure 9 et conduisent à des distributions quasi-hyperboliques des pressions p mesurées (Fig. 9.a) et des rapports $k=p / u$ (c'est-à-dire le coefficient de réaction, Fig. 9.b) en fonction des déplacements u mesurés. Il y a lieu de noter qu'un tel résultat est plausible car la raideur du sol ne peut, à l'instar du module de déformation, que diminuer lorsqu'il se produit un « relâchement $»$ des grains du sol (Rowe, 1962) derrière la paroi par rupture en butée ou en poussée.

Pour apprécier l'incidence des divers paramètres sur les déplacernents u (en tête) de la paroi autostable, on a porté sur la figure 10 les déplacements (normalisés par la valeur du déplacement maximal) en fonction des valeurs courantes de paramètres, $\mathrm{k}_{\mathrm{h}}$, cohésion $\mathrm{c}$, angle de frottement interne $\varphi$ et rigidité propre de la paroi, (normalisés par la valeur maximale du paramètre

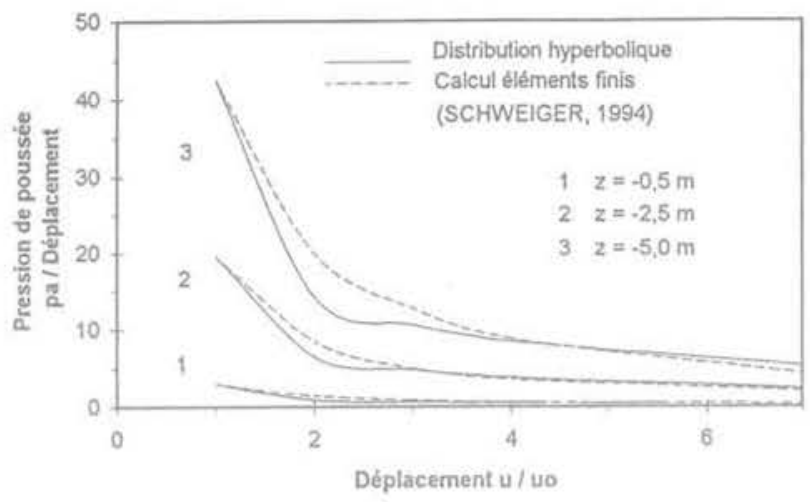

FG.8 Coefficient de réaction en fonction du déplacement pour une paroi rigide soumise à des déplacements de translation (H.F. Schweiger, 1994).

Subgrade reaction coefficient versus displacement for a stiff retaining wall submitted to translation.

d'étude). La représentation adimensionnelle de la figure 10 nous conduit à préciser les points suivants :

- le coefficient de réaction $k$, est de près le paramètre le moins influent sur les variations des déplacements : au-delà d'une valeur caractéristique donnée, une augmentation de $k_{b}$ de $100 \%$ entraîne une diminution des déplacements de la paroi autostable de $15 \%$;

- l'incidence des paramètres de cisaillement $c$ et $\varphi$ est totalement différente : en effet, les déplacements présentent des variations plus homogènes et régulières quel que soit l'angle de frottement interne du sol, par contre, la cohésion conduit à une stabilisation des déplacements à partir d'une valeur caractéristique donnée alors que dans la plage des faibles valeurs de la cohésion c, les déplacements subissent des variations importantes ;

- les variations de la rigidité propre de la paroi conduisent, comme la cohésion, à d'importantes fluctuations des déplacements. Cependant, ces variations semblent se stabiliser également pour une valeur caractéristique donnée de la rigidité.
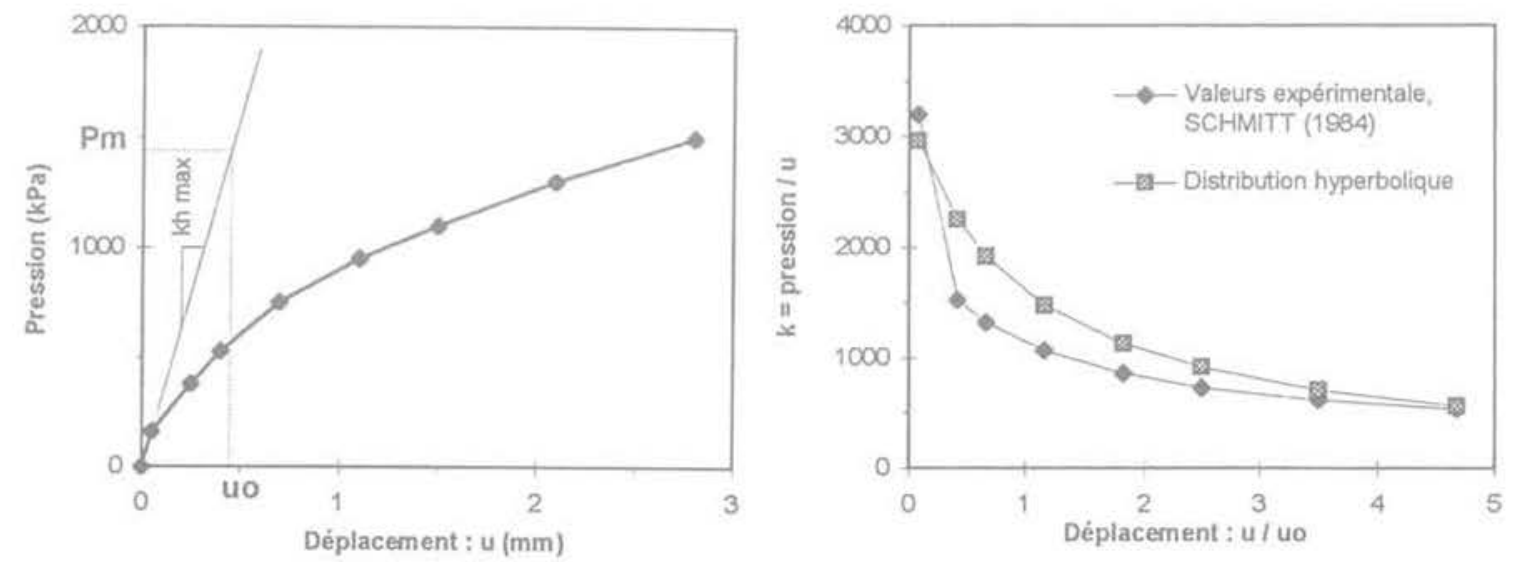

Fic.9. Variations expérimentales des pressions et des coefficients de réaction en fonction du déplacement pour une paroi tirantée (métro de Lille, P. Schmitt, 1984).

Pressure and subgrade reaction coefficient versus displacement for an anchored retaining wall : experimental results (métro de Lille, P. Schmitt, 1984). 

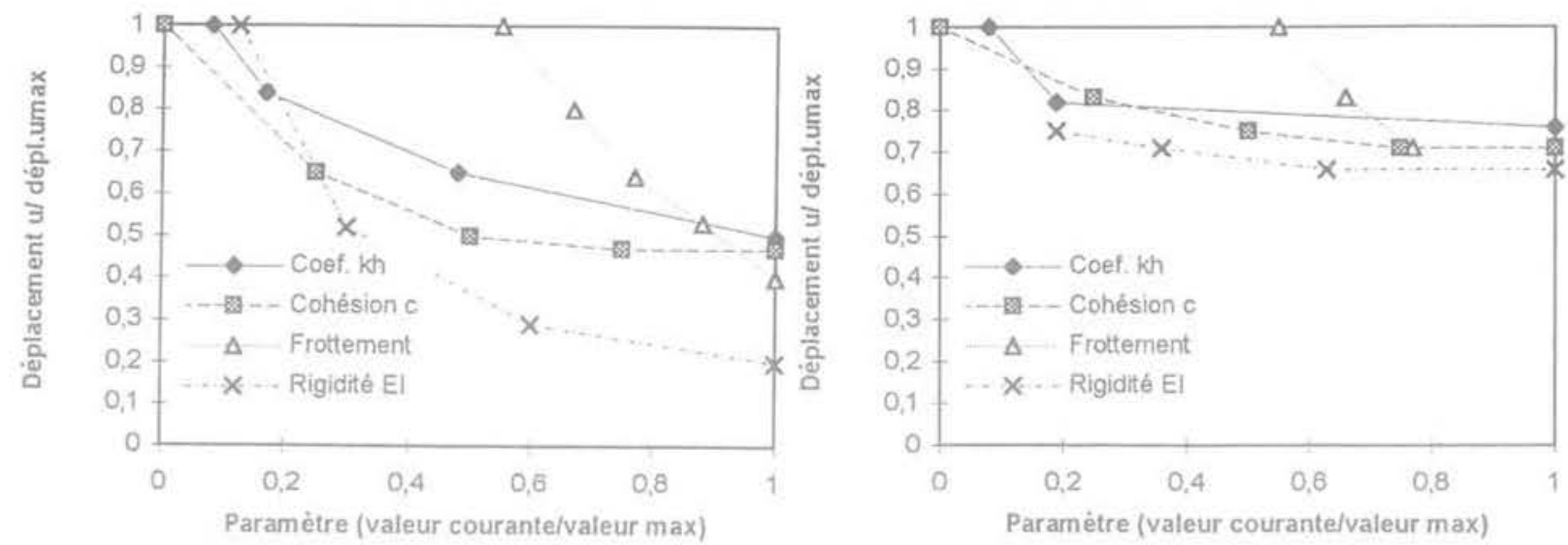

$$
\text { a - Parol auto-stable b-Parol avec tirants }
$$

FG.10 Incidence des divers paramètres sur les variations des déplacements de la paroi autostable. Parameters variation effect of self-stable retaining wall displacements.

En somme, l'étude paramétrique abordée ici montre que l'influence des tirants d'ancrage, de la cohésion du sol et de la rigidité propre de la paroi est la plus importante sur l'évolution des déplacements et des pressions derrière la paroi.

\section{3}

\section{Conclusion}

Cette étude avait pour but d'analyser, à partir du comportement " parfaitement élastique » d'un ressort (modèle de Winkler) l'analogie "sol-ressort ) adoptée ici pour expliciter la notion de coefficient de réaction utilisée en France dans les codes automatiques de calculs et de dimensionnement des ouvrages de soutènement.

Une telle analogie montre que la raideur du ressort " régit $),$ cormme le coefficient de réaction du sol (sol ( assimilé » à un ressort), le domaine des déplacements d'origine élastique ou « pseudo-élastique s : plus la raideur du matériau (sol ou ressort) est grande, moins les déplacements sont élevés et plus la rupture est probable.

L'examen des formulations, utilisées en France sur la base des résultats pressiométriques pour évaluer les coefficients de réaction pour des cas de type semelle ou paroi de soutènement, montre que le coefficient de réaction n'est pas un paramètre intrinsèque du sol et que les méthodes aux coefficients de réaction ne constituent pas des lois d'interaction sol-structure de contact puisque même tout en assurant la continuité des déplacements normaux, elles ne respectent pas, dans leur forme actuelle, la continuité des déplacements tangentiels.

L'approche de type A. Monnet est fort intéressante puisqu'elle intègre à la fois la rigidité El de la paroi et les caractéristiques de cisaillement c et $\varphi$ des sols. L'introduction de la rigidité de la paroi est, à notre avis, indiscutable car une paroi rigide conduit à une diminution des déplacements et à des augmentations des contraintes derrière la paroi rigide, augmentations qui ne peuvent pas être compensées par la limitation des déplacements, le coefficient de réaction ne peut qu'augmenter. Cependant, cette formulation présente l'inconvénient de ne pas tenir compte de la raideur propre du sol puisque, à titre d'exemple, une telle formulation conduit à une même valeur du coefficient de réaction pour un sable à compacité moyenne que pour un limon possédant, à long terme, le même angle de frottement.

L'étude paramétrique effectuée montre que le coefficient de réaction ne présente d'incidence que sur les états élastiques ou (" pseudo-élastiques ») du sol, donc en pratique sur les premières phases de terrassement. Dans ces phases, le coefficient de réaction dépend effectivement de la raideur du sol et de ses caractéristiques de cisaillement ainsi que de la rigidité propre de la paroi et de la rigidité relative sol-structure de contact. Néanmoins, les méthodes aux coefficients de réaction utilisées dans les codes de calculs automatiques ( $($ DENEBOLA-LPC », « RIDO », «PAROI ») conduisent à des variations des déplacements et des pressions résultantes en fonction du coefficient de réaction généralement stabilisées pour un paramètre caractéristique donné (cohésion c, rigidité El, éléments de confortement tels que tirants ou butons).

L'inconvénient de ces méthodes réside dans le choix d'une loi de réaction linéaire entre déplacements et pressions mobilisés qui conduit à l'estimation d'un coefficient de réaction constant quel que soit le niveau de déplacements atteint de la structure de soutènement.

Le choix d'une loi de réaction hyperbolique pouvant pallier de telles carences nécessite des résultats expérimentaux qui permettent de valider de telles lois théoriques, les études à venir ne devront pas être destinées uniquement, à notre avis, à déterminer la « bonne x valeur du coefficient de réaction à prendre dans les calculs des parois de soutènement mais plutôt à chercher la loi de réaction qui s'adapte le mieux au comportement non linéaire du sol.

\section{Remerciements}

M. Michel Gambin a accepté avec beaucoup de gentillesse et de dévouement de lire, d'apporter d'amples et de précieux conseils et de relire le présent texte. L'auteur tient à le remercier très vivement. 
Balay J. - Recommandations pour le choix des paramètres de calcul des écrans de soutènement par la méthode aux modules de réaction. Fiche de recherche LCPC, 1985, 24 pages.

Balay J., Frank R., Harfouche L, - Programme "DENEBOLA " pour le calcul des soutènements par la méthode des modules de réaction, Bull. Liaison LPC 120, juillet-août 1974, p. 3-12.

Barbas A., Frank R, - Utilisation des éléments finis en Mécanique des Sols dans le domaine de l'élasto-plasticité. Rapport de recherche LCPC $n^{\circ} 116,1982$.

Barussaud S. - Ouvrages de soutènement Étude théorique de l'interaction solparoi-ancrage, CA LREP, 1980-1981.

Gigan 3.-P. - Expérimentation d'un rideau de palplanches ancré par tirants actifs, Bulletin de Liaison des LPC 129, janvierfévrier 1984, p. 5-20.

Josseaume H. - Méthode de calcul des rideaux de palpianches : étude biblio. graphique, Bull. Liaison LPC 72, juilletaoût 1974, p. 177-212.
Kondner B.I - Hyperbolic Stress-strain Response : Cohesive Soils, Journal of the Soil Mechanics and Foundations Division, ASCE, vol. 89, n SM1, Proc. Paper 3429, 1963.

Ménard L., Bourdon G. - Calcul des rideatux de soutènement: méthode nouvelle prenant en compte les conditions réelles d'encastrement, Sols-Soils 12. 1965, p. 18-32.

Ménard I., Bourdon G., Houy A. - Etude expérimentale de l'encastrement d'un rideau en fonction des caractéristiques pressiométriques du sol de fondation, Sols-Soils 9, 1964, p. 11-27.

Mẻnard L., Bourdon G., Gambin M. Méthode générale de calcul d'un rideat ou d'un pieu sollicité horizontalement en fonction des résultats pressiométriques, Sols-Soils 22-23

Monnet A. - Module de réaction, coefficient de décompression, au sujet des paramètres utilisés dans la méthode de calcul élasto-plastique des soutène- ments, Revue française de Géotechnique 65, 1994, p. 65-72

Rowe P.W. - Sheet-pile walls in clav, Institution of Civil Enginers, Proceedings, vol. 7, London, 1957.

Rowe P.W. - The stress-dilatancy relation for static equilibrium of an assembly of particules in contact. Proc, of the Royal Society, Series A, 269, 1962, 500-527.

Schmitt P. - Étude expérimentale de la sollicitation exercée par le sol sur les ouvrages de soutènement simples, Revue française de Géotechnique n ${ }^{\circ} 28$, 1984.

Schweiger H.F. - On the use of DruckerPrager failure criteria for earth pressure problems, Computers and Geotechnics 16, 1994, p. 223-246

Terzaghi K. - Evaluation of coefficients of subgrade reaction, Geotechnique vol. 4 , Londres, 1955.

Winkler E. - Die Lehre von der Elasticităt und Festigkeit, Prag. Verlag H. Dominicus, 1868 